\title{
Modelación de la producción de metano en el Relleno Sanitario Parque Ambiental Palangana (Santa Marta)
}

\section{Modelling of Methane Production in "Parque Ambiental Palangana" Landfill (Santa Marta)}

\author{
Armenta-Rivas Maysson Esmi \\ Grupo de Investigación en Modelación de Sistemas Ambientales, Colombia \\ Correo: may.armen61@gmail.com \\ Sierra-Camargo Liseth Dayana \\ Grupo de Investigación en Modelación de Sistemas Ambientales, Colombia \\ Correo: lisi1502@gmail.com
}

Vélez-Pereira Andrés M.

Grupo de Investigación en Modelación de Sistemas Ambientales, Colombia

Correo: avelezpereira@gmail.com

\section{Resumen}

El metano generado en rellenos sanitarios se puede presentar como un problema ambiental en la gestión de los residuos sólidos, pero también se considera como un beneficio por su alto potencial energético. El objetivo del estudio fue estimar la producción de metano en el Relleno Sanitario Parque Ambiental Palangana de Santa Marta-Colombia; aplicando los modelos matemáticos del Panel Intergubernamental de Cambio Climático (IPCC) y Corenostós, empleando la deposición mensual de residuos sólidos, su composición físico-química y las condiciones meteorológicas desde 2004 al 2013 y proyectadas hasta el 2019 como periodo de vida útil. Los resultados muestran que la ciudad posee una alta producción per-cápita de residuos $(0.83 \mathrm{~kg} / \mathrm{hab}$-día), con un alto contenido de humedad y compuesta principalmente por materia orgánica (39.7\%). El modelo del IPCC estimó una máxima producción de 2949 Ton/año de metano un año posterior al cierre estimado, y un error promedio de estimación de 18.4\%; mientras que el modelo Corenostós estimó una máxima producción de 1593 Ton/año de metano en el año de clausura estimado, con un error promedio de 47.2\%. Ambos modelos presentan una sub-estimación de las emisiones cuantificadas en el relleno, debido a las condiciones particulares de operación y al alto contenido de humedad de los residuos; no obstante, el modelo del IPCC presenta un error aceptable para realizar las estimaciones de metano en el relleno.

Descriptores: biogás, Corenostós, metano, modelos de estimación, relleno sanitario, residuos sólidos urbanos.

\begin{abstract}
Methane generated in landfills could be considered as an environmental problem in solid waste management, but could also be profitable for its high energy potential. The aim of the study was to estimate methane production in the "Parque Ambiental Palangana" landfill (Santa Marta-Colombia). There were applied the mathematical models of the Intergovernmental Panel on Climate Change (IPCC) and Corenostós, by using the information of monthly deposition and physical-chemical composition of solid waste and meteorological conditions since 2004 to 2013 and projected ones to 2019 as useful life. The results showed that the city has a high per-capita solid waste production $(0.83 \mathrm{~kg} /$ inhabitant-day), with high moisture content and mainly composed of organic matter (39.7\%). The model of the IPCC estimated a maximum production of 2949 Tons/yr of methane, a year later than estimated closing and an average error of $18.4 \%$; while Corenostós model estimated a maximum production of 1593 Tons/ yr of methane in the year of estimated closing, with a $47.2 \%$ of error. Both models showed an underestimation according to data emission presented by the landfill, due to the particular operating conditions and the high moisture content of the solid waste, however the IPCC model has an acceptable error to estimate methane in the landfill.
\end{abstract}

Keywords: biogas, Corenostós, methane, estimation models, landfill, urban soild waste. 


\section{INTRODUCCIÓN}

La producción de residuos sólidos municipales (RSM) ha experimentado un crecimiento progresivo durante las últimas décadas como consecuencia del aumento poblacional; ya que existe una relación directa entre la generación y caracterización de residuos, con la cantidad de habitantes, tamaño y expansión de las ciudades (Arrieta, 2008; Jaramillo y Zapata, 2008; Singh et al., 2011; Rojas et al., 2014). Se consideraba que más de la mitad de los desechos producidos eran de origen orgánico, y solo $2 \%$ recibían un adecuado tratamiento para su aprovechamiento; el porcentaje restante se distribuía entre rellenos sanitarios y otros tipos de disposición. Actualmente los RSM poseen una cantidad mayor de plásticos, papeles y materiales reutilizables que desechos orgánicos debido al crecimiento de áreas metropolitanas (Jaramillo y Zapata, 2008; Terraza, 2009; Hoornweg y Bhada-Tata, 2012), afectando la vida útil de los rellenos, al tiempo que se deprecia el valor comercial de estos materiales susceptibles de aprovechar.

La disposición de RSM en el relleno sanitario (RESA), incrementa las emisiones de biogás, producto de la degradación de los residuos orgánicos en condiciones anaerobias (Georgaki et al., 2008; Hilkiah-Igoni et al., 2008; Im et al., 2009; Vera-Romero et al., 2015). El biogás, está conformado por trazas de compuestos orgánicos volátiles (COV), sulfuro de hidrogeno $\left(\mathrm{H}_{2} \mathrm{~S}\right)$ y principalmente por dióxido de carbono $\left(\mathrm{CO}_{2}\right)$ y metano $\left(\mathrm{CH}_{4}\right)$, este último, relevante por sus efectos en la regulación térmica de la tierra, pues posee un poder 21 veces mayor de retención de radiación solar terrestre comparado con el dióxido de carbono (Meraz et al., 2004; Ducom et al., 2009; Im et al., 2009; Sierra et al., 2014). Se calcula que $14 \%$ de las emisiones globales de $\mathrm{CH}_{4}$ se relacionan con los rellenos sanitarios (Martín, 1997; Kim y Yi, 2009; Aguilar-Virgen et al., 2011a), convirtiendo estos en una fuente importante de emisiones de gases de efecto invernadero (GEI) a la atmosfera. Por lo que las estimaciones de la producción de biogás en los RESA, se establecieron en una obligación para muchos países comprometidos con el objetivo del protocolo de Kioto de disminuir las emisiones de gas metano (Aronica et al., 2009; Mackie y Cooper, 2009).

Otros impactos de la producción de metano en los RESA, son las emisiones fugitivas desde la superficie; ya que puede generar un desplazamiento del oxígeno en el material de cobertura afectando el crecimiento de las raíces de árboles, arbustos o pasto que se implementen en la etapa de clausura. Asimismo, se establece el riesgo latente de explosión si la concentración volumétrica de $\mathrm{CH}_{4}$ contenido en el biogás, es igual o superior a 5\% (Schmidt, 1999; SCS Engineers, 2008; Serrano, 2006).

La estimación de metano generado en el relleno se puede realizar mediante modelos matemáticos, los cuales evalúan la dinámica de generación de biogás, incorporando variables como la fecha de apertura y clausura, constantes de degradación biológica y físico-química entre otras, que permiten describir con alto nivel de precisión la generación de biogás dentro del relleno (Lobo y Tejero, 2007; Guerra, 2009; Cendales, 2011, Kalantarifard y Yang, 2012). El modelo del IPCC (Kim y Yi, 2009; Wangyao et al., 2010), el LandGEM (Panesso et al., 2011; Kalantarifard y Yang, 2012; Vera-Romero et al., 2014) y el modelo mexicano de biogás (Aguilar-Virgen et al., 2011b; 2011a) se establecen como algunos de los modelos frecuentemente aplicados.

La cuantificación de metano por medio de modelos permite que su captura y uso como fuente de energía renovable se convierta en uno de los principales mecanismos de desarrollo limpio en los RESA, puesto que el $\mathrm{CH}_{4}$ presenta un poder calorífico entre 400 y 600 BTU, equivalente a la mitad del gas natural (Murphy y McKeogh, 2006; Zamorano et al., 2007; Espíritu-Barragán et al., 2014); permitiendo su transformación en energía calórica para la generación de electricidad, como fuente de calor en sistemas de calefacción, combustible de caldera, combustible de vehículos, entre otros usos, que reducirían significativamente las emisiones de GEI.

En la ciudad de Santa Marta, la población se ha incrementado a una tasa de $3.16 \%$, producto de migraciones de diferentes zonas del país y del departamento del Magdalena; aumentado la producción de RSM de 320 Ton/día en 2008 a 410 Ton/día en 2011 (Aluna consultores limitada, 2011; SSPD Superintendencia de servicios públicos, 2011; Alcaldía de Santa Marta et al., 2012). Teniendo en cuenta que la estimación de la producción de biogás en los RESA se considera una estrategia ambiental y económica importante, enfocado en el aprovechamiento tecnológico de las cantidades de metano generado, por su potencial como fuente de energía no convencional. El objetivo del presente documento fue estimar la producción de $\mathrm{CH}_{4}$ en el Relleno Sanitario Parque Ambiental Palangana ubicado en la ciudad de Santa Marta-Colombia. Para ello se aplicaron los modelos matemáticos del Panel Intergubernamental de Cambio Climático (IPCC) y Corenostós, empleando como variables de entrada la deposición mensual de residuos sólidos en el RESA, su composición físico-química y las condiciones meteorológicas. 


\section{Metodología}

\section{ÁREA DE ESTUDIO}

El relleno sanitario del distrito de Santa Marta está localizado en el sector denominado Palangana, del que recibe su nombre, cuenta con un área de 53 ha. Se encuentra ubicado aproximadamente a $1.100 \mathrm{~m}$ del casco urbano sobre la vía que conduce a Bahía Concha (Parque Nacional Natural Tayrona) y próximo a los habitantes de las comunidades que residen en la urbanización Altos de Bahía Concha y los barrios Fundadores y Bastidas (Vélez-Pereira et al., 2010; Camargo et al., 2011). Es un relleno tipo terraza que inició operaciones en el mes de julio del 2004 y cuenta con una capacidad de 2.156.675 Ton (ESPA, 2014). La temperatura media varía entre 28$32{ }^{\circ} \mathrm{C}$, con precipitaciones anuales de $500-1000 \mathrm{~mm} /$ año (Garrido y Camargo, 2015).

\section{VARIABLES DE ENTRADA DEL MODELO}

\section{CUANTIFICACIÓN Y COMPOSICIÓN DE RESIDUOS DEPOSITADOS EN EL RELLENO}

La información de las cantidades, composición química y física de los residuos depositados en el RESA durante el periodo 2004-2013, provienen de los informes de calidad ambiental (ICA), los cuales se presentan anualmente por la empresa prestadora del servicio a la autoridad ambiental. Posteriormente se realizaron las proyecciones de disposición de residuo mediante el método geométrico hasta el año de clausura (2019), como fecha estipulada en el plan de gestión de residuos sólidos (PGIRS) de Santa Marta con el fin de alimentar los modelos.

\section{CONDICIONES METEOROLÓGICAS}

La información meteorológica proviene de la estación Núm. 15015050-Aeropuerto Simón Bolívar, ubicada a 11.07 de latitud norte, 74.13 longitud oeste y 4 m.s.n.m. en la ciudad de Santa Marta. De ella se recopilaron el valor medio mensual multianual de la temperatura media $\left({ }^{\circ} \mathrm{C}\right)$, evaporación total $(\mathrm{mm})$ y precipitación total (mm) para el periodo de estudio (2004-2013), posteriormente se realizaron las proyecciones por el método de la razón normal hasta 2034, 15 años después del cierre proyectado, reportado como el tiempo de eversuria para los RESA (Monsalve, 1999).
ESTIMACIÓN DE LA PRODUCCIÓN DE METANO POR LOS MODELOS

Para la cuantificación de metano se aplicaron dos modelos: el modelo IPCC (IPCC, Panel Intergubernamental de Cambio Climático, 1996) y el modelo de Corenostós (Collazos, 2005). Las particularidades de aplicación para cada modelo se describen a continuación.

\section{MODELO IPCC}

El método de la IPCC (Tier2) se basa en un balance de masa, en el cual la estimación de la producción de biogás depende de las categorías de residuos, el carbono orgánico degradable y el gas metano en el relleno. (IPCC, Panel Intergubernamental de Cambio Climático, 1996; Kim y Yi, 2009; Aguilar-Virgen et al., 2011b; 2012).

Las ecuaciones matemáticas mediante las que se rige el modelo de primer orden de la IPCC son las ecuaciones 1 y 2

$$
\begin{aligned}
& E_{C H 4}=\left(\left\{A * k^{*} R S U_{T}{ }^{*} R S U_{F}{ }^{*} F M C{ }^{*} L_{o}{ }^{*} e^{-k(T-x)}\right\}-R\right) * \\
& (\mathbf{1}-\mathrm{OX}) \\
& L_{o}=F M C * C O D * C O D_{F}^{*} F * \frac{16}{12}
\end{aligned}
$$

donde

$E_{\mathrm{CH}^{4}}=$ emisión de metano en Ton/año

$A=$ factor de normalización para la corrección de la sumatoria

$R S U_{T}=$ total de residuos sólidos municipales generados (G/año)

$R S U_{F}=$ fracción o categoría de dichos residuos (Gg/año)

$L_{o}=$ potencial de generación de metano $\left(\mathrm{m}^{3} / \mathrm{Mg}\right.$ de residuos)

$k=$ índice de generación de metano

$T=$ año en transcurso

$x=$ año de entrada de los residuos

$F=$ fracción de $\mathrm{CH}_{4}$ en el gas de vertedero (se asume un valor de 0.5 , pues el biogás se compone gene ralmente por $50 \%$ de gas $\mathrm{CH}_{4}, 50 \%$ de $\mathrm{CO}_{2}$ y menos de $1 \%$ de otras trazas constituyentes)

$\boldsymbol{R}=\mathrm{CH}_{4}$ recuperado $(\mathrm{G} / \mathrm{año})$

$O X=$ factor de oxidación (valor por defecto es 0)

$F C M=$ factor de corrección para el metano, un ajuste de la estimación de biogás en el modelo que tiene en cuenta el grado de degradación anaerobia de los resisudos, 
su valor está relacionado con la profundidad y tipo de relleno con el que se cuente.

El COD es el carbono orgánico degradable, que depende de la composición de los residuos y puede calcularse a partir del porcentaje promedio de carbono degradable presente en los componentes de los residuos, utilizando la ecuación 3.

$C O D=0.4(F)+0.17(B)+0.15(C)+0.30(D)$

donde

$F=$ porcentaje de residuos de papel, cartón y textiles

$B=$ residuos de desecho de jardín, parques o desechos orgánicos putrescibles (excluidos los ali mentos)

$C=$ porcentaje de los residuos que corresponde a restos de alimentos

$D=$ porcentaje de residuos que corresponde a madera y paja.

$C O D_{F}$ es la fracción de carbono orgánico degradable asimilado o que se convierte en biogás, el valor predeterminado utilizado por el IPCC es de 0.77. Sin embargo, este puede variar de 0.42 para $10^{\circ} \mathrm{C}$ a 0.98 para $50^{\circ} \mathrm{C}$. Su cálculo se basa en la temperatura que se maneja en la zona anaerobia del RESA.

$C D O_{F}=0.014 T+0.28$

\section{Modelo Corenostós}

El modelo Corenostós simula el proceso de llenado de un relleno sanitario, calculando los gases y lixiviados que se van produciendo por la biodegradación de los desechos orgánicos dispuestos y por la infiltración de las lluvias, que llegan a tener contacto directo con los lechos de desechos en el RESA (Collazos, 2005).

$$
\mathrm{C}_{c} \mathrm{H}_{h} \mathrm{O}_{o} \mathrm{~N}_{n}+\mathrm{WH}_{2} \mathrm{O} \rightarrow \mathrm{XCH}_{4}+\mathrm{YCO}_{2}+\mathrm{ZNH}_{3}
$$

El modelo basa su fundamento teórico en una ecuación empírica (ecuación 5), donde el porcentaje de residuos putrescibles, papel o cartón, textiles y jardinería, así como el porcentaje de humedad se se expresa en la composición química de carbono, hidrógeno, oxígeno, nitrógeno y azufre; estableciendo un balance y por ende una estimación del metano producido.

\section{MÉTOdOS ESTADÍSTICOS}

El grado de precisión de los modelos aplicados se estableció mediante el error medio absoluto (MAE) y la raíz media del error cuadrático (RMSE). Los valores se compararon estadísticamente mediante un análisis de varianza simple (ANOVA) para determinar si existe una diferencia o no significativa entre las estimaciones y los valores simulados. Adicionalmente, se aplicó la prueba no paramétrica de Wilcoxon-Mann-Whitney (Wilcoxon, 1945; Mann y Whitney, 1947) para establecer el nivel de significancia de las diferencias.

\section{DisCUSIÓN Y ANÁLISIS DE RESULTADOS}

\section{DISPOSICIÓN Y COMPOSICIÓN DE RESIDUOS SÓLIDOS EN EL RESA}

En la figura 1 se observa el comportamiento que registra la disposición de los residuos sólidos en el RESA Palangana durante los años de operación 2004-2013. En los meses de enero, noviembre y diciembre se registraron picos de mayor disposición, con valores promedios de 10846 Ton/mes, 11007 Ton/mes y 11830 Ton/mes, respectivamente, donde el mes de diciembre es el que presenta mayor generación de residuos sólidos a lo largo de los datos históricos, consecuencia de las migraciones por periodos vacacionales que incrementan la producción de residuos y por ende la producción per cápita (ppc) en la ciudad $(0.83 \mathrm{~kg} / \mathrm{hab}-$ día); la cual es mayor que otras ciudades con mayor población como Medellín (0.591 kg/hab-día) que cuadriplican en número de habitantes a Santa Marta (Observatorio de Políticas Públicas de Medellín, 2010). Incluso la ppc de Santa Marta se reporta por encima del estándar colombiano establecido en el RAS de 0.79 kg/hab-día (Ministerio de Vivienda, Ciudad y Territorio y Viceministerio de Agua y Saneamiento Básico, 2012).

En Santa Marta, los residuos se componen en su mayoría, por residuos de jardín y orgánicos (figura 2). Estas proporciones se han mantenido constantes durante toda la vida útil del RESA (ESPA, 2014), por tanto, las toneladas dispuestas por cada tipo de residuo aumentan conforme a la tasa de generación de residuos estimada en $3.76 \%$ anual. La distribución por tipo de residuo es consistente con las actividades económicas del distrito: turismo, pesca, comercio, actividad portuaria y producción de productos de base agrícola. Por otro lado, la proporción de residuos orgánicos del distrito (47\%) es un poco mayor a la registrada en otros países como India (38.6\% de los residuos 


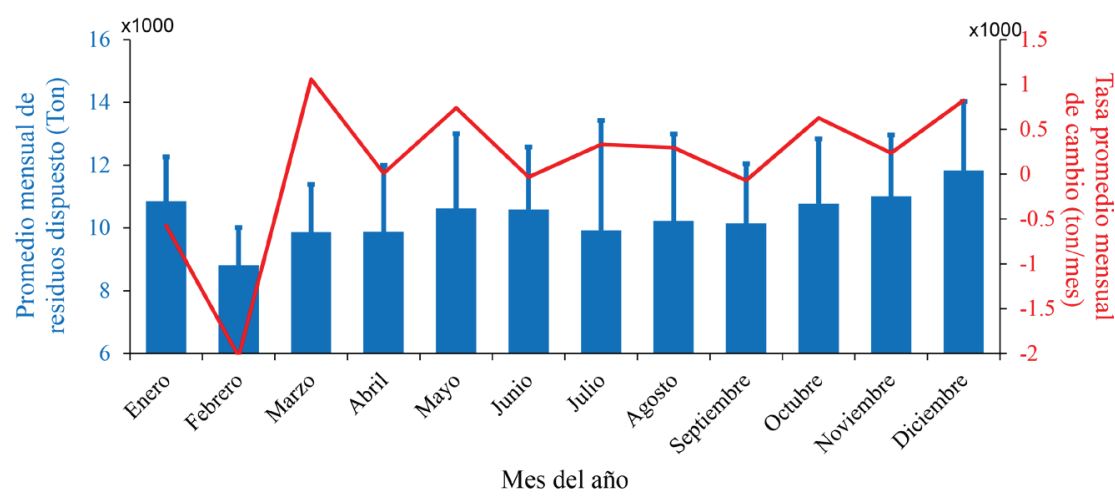

Figura 1. Promedio y tasa de cambio mensual multianual de los residuos depositados en el RESA Parque Ambiental Palangana son orgánicos), México (36.20\%), Brasil (30\%) (SCS Engineers, 2005; 2008; Serrano, 2006; ESPA, 2014; VeraRomero et al., 2014); pero para el contexto colombiano, se reporta dentro de los valores registrados en ciudades como Cali (75\%), Bucaramanga (50.3\%), Bogotá $(65 \%)$. Este alto porcentaje de putrescibles es directamente proporcional a la generación de biogás y metano; debido a que la descomposición anaerobia que produce biogás en los rellenos, depende en gran parte del contenido orgánico (desecho de jardín, comida y papel que se generan en el hogar) que se dispone en él (Meraz et al., 2004; Serrano, 2006).

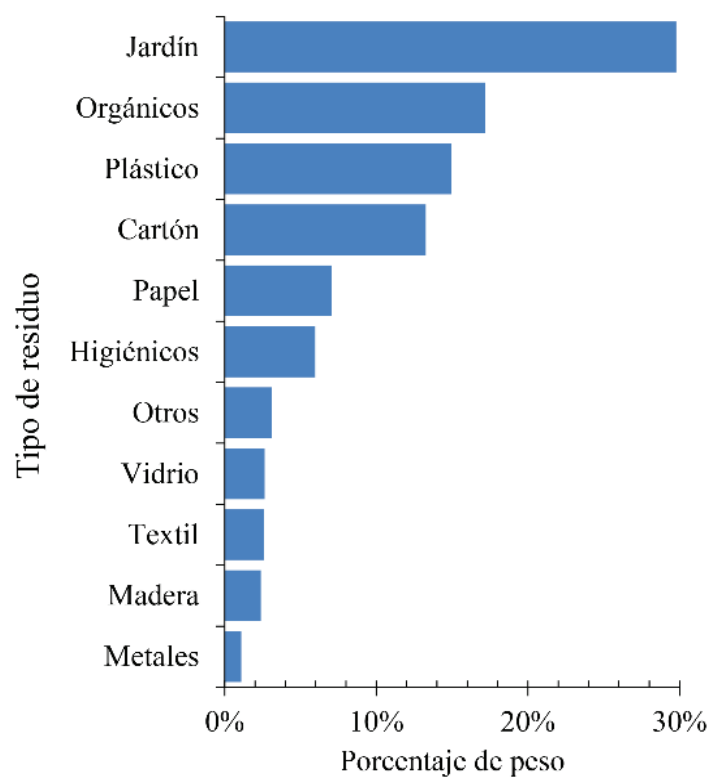

Figura 2. Distribución porcentual por tipo de residuo depositado en el RESA Parque Ambiental Palangana

\section{ESTIMACIÓN DE LAS EMISIONES POR LOS MODELOS}

Los modelos IPCC y Corenostós se comportan de forma similar (figura 3), presentando crecimiento exponencial de la producción de metano hasta el año de clausura del RESA (año 2019), donde se detiene la disposición de residuos sólidos. La mayor generación de $\mathrm{CH}_{4}$ se evidencia en este año para el modelo Corenostós, con una emisión de 1593 Ton/año de $\mathrm{CH}_{4}$; mientras que el IPCC tiene su punto máximo en el año inmediatamente posterior al cierre del RESA (año 2020) con 2.949 Ton/año de $\mathrm{CH}_{4}$. Esto es coherente con Aguilar-Virgen et al. (2011a) quienes aseguran que después del cierre, la emisión del metano empieza un decrecimiento hasta estabilizarse o cesar. En nuestro caso, el modelo del IPCC se estabilizaría en el 2053 con 409 Ton/año; al mismo tiempo, el modelo Corenostós fija este periodo en el año 2034 con una emisión de 40 Ton/año de metano.

Por otro lado, también se puede observar que la estimación del modelo del IPCC es 1.63 veces mayor a la del modelo Corenostós, antes de la clausura del relleno. Posterior a ella, el Corenostós tiene un decaimiento más acelerado mientras el modelo del IPCC continúa estimando emisiones. Estas diferencias pueden relacionarse con las variables asumidas en cada modelo; en el caso del modelo Corenostós se manejaron valores únicos para la composición química y el índice de generación de metano (k), sin tener en cuenta las condiciones climáticas o zonas donde se ubica el RESA, ya que solo contempla la precipitación como una variable de importancia en la generación de lixiviado. Además, asume que la generación de gases se produce en el mismo año en que los residuos se depositan y que la mayor degradación aparece en los primeros cinco años (Collazos, 2005). Mientras que el modelo del IPCC, cuenta con valores de acuerdo con la ubicación geográfica; adicionalmente, la estimación 


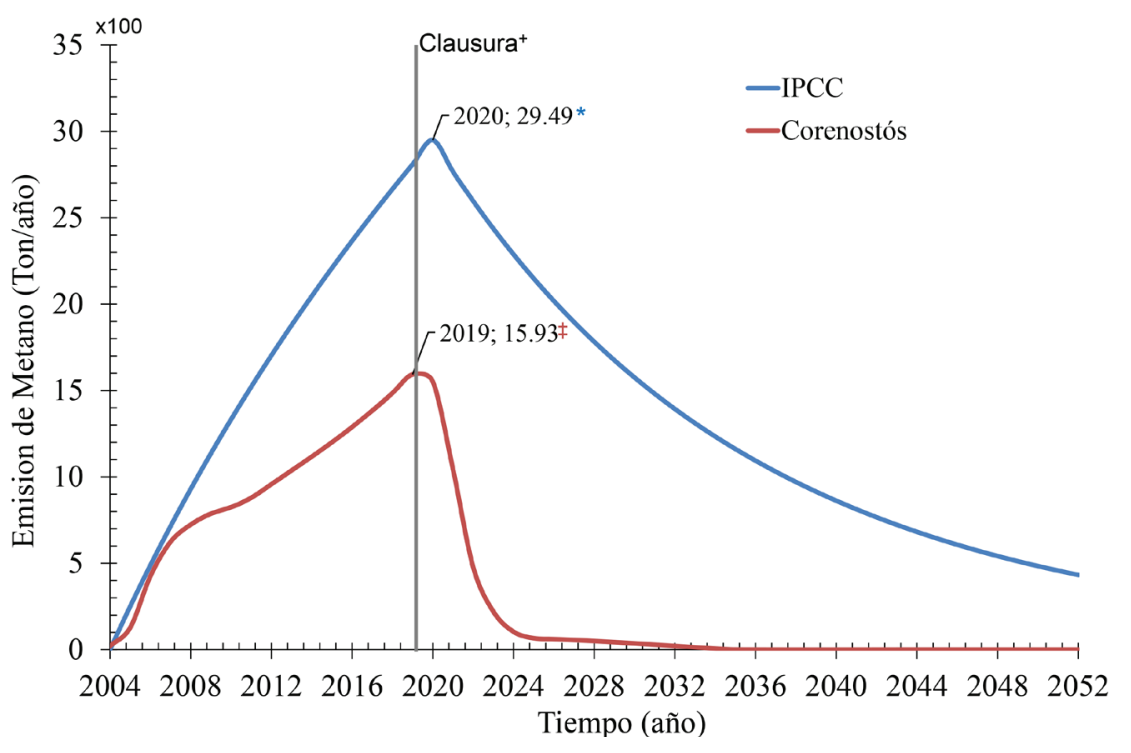

Figura 3. Variación de la producción de Metano estimado por los modelos

${ }^{+}$Fecha estimada de clausura del relleno sanitario. *Año y máxima producción de metano estimada por el modelo del IPCC.

${ }^{\ddagger}$ Año y máxima producción de metano estimada por el modelo del Corenostós se establece a partir de la población, la producción per-cápita y el índice de generación de metano $(\mathrm{k})$ por cada categoría de residuos que ingresan al RESA (IPCC, Panel Intergubernamental de Cambio Climático, 1996; Kim y Yi, 2009; López, 2011; Aguilar-Virgen et al., 2011b; 2012), donde la cantidad por tipo de residuo es la variable que puede generar el mayor grado de incertidumbre en las estimaciones, si no se tiene un precisión de la variación en la composición de los residuos mes a mes o año con año.

En la figura 4 se observa que las estimaciones del modelo Corenostós están muy alejadas de los valores reales reportados en los ICA; mientras las estimaciones del IPCC se aproximan mejor a ellos. Esto se evidencia en los valores del error calculado, donde el Corenostós supera un $29 \%$ el error calculado del modelo IPCC. Estos resultados se confirman por el ANOVA, que reporta una diferencia entre los valores estimados y medidos (P-valor: 0.024); no obstante, solo para el caso del modelo Corenostós, la prueba de Wilcoxon-Mann-Whitney estima que la diferencia es estadísticamente significativa a $95 \%$ con el valor medido y la estimación establecida por el IPCC.

En general, se observa que los modelos están subestimando la producción de metano, incluso esta diferencia podría llegar a ser mayor si se tienen en cuenta las emisiones fugitivas en la superficie del relleno, que no son cuantificables en las mediciones periódicas que realiza el relleno en las chimeneas. Las diferencias se pueden atribuir al alto contenido de humedad $(29.80 \%)$ de los residuos sólidos depositados, aunado a la abundante precipitación del área de estudio y las posibles prácticas de recirculación de lixiviado por as- persión, que generan la reducción del tiempo transcurridos en las fases anaerobia y metanogénica; situación que no se asimila por los modelos y permite un aumento en la producción del metano (Camargo y VélezPereira, 2009; Rada et al., 2015). Otra razón particular por la que el modelo IPCC presenta esta subestimación, es la alta fluctuación intermensual que presenta la ppc, la cual no se puede suministrar al modelo para disminuir el grado de incertidumbre en las estimaciones (IPCC, Panel Intergubernamental de Cambio Climático, 2002; 2006).

Los resultados del error cuadrático medio (RMSE) muestran que para el modelo IPCC es de $\pm 23.4 \%$ con relación a las mediciones presentadas en los ICA; por su parte, el Corenostós exhibe un error de $\pm 51.7 \%$ frente a los valores medidos. Este último valor de error es mayor al obtenido por Mejía y Gil (2015) de 33.5\% y mucho menor al reportado por Urrego-Martínez et al. (2016) en el relleno sanitario Doña Juana, Colombia. En consecuencia, las estimaciones arrojadas por el método IPCC se ajustan mejor a las mediciones realizadas en el RESA Palangana. El error del IPCC se puede considerar aceptable, pues se encuentra dentro de un rango de significancia media-alta, en comparación con los estudios realizados en Cuba $( \pm 36.1 \%)$ y Suecia $( \pm 9 \%)$ que presentan una alta diversidad de condiciones en las áreas analizadas (Börjesson et al., 2009; López, 2011), y se corrobora por el test de WilcoxonMann-Whitney que no reporta una diferencia significativa entre las estimaciones y las medianas de metano en el RESA Parque Ambiental Palangana. Finalmente se establece como necesario para mejorar la precisión en las estimaciones y disminuir el grado de incerti- 


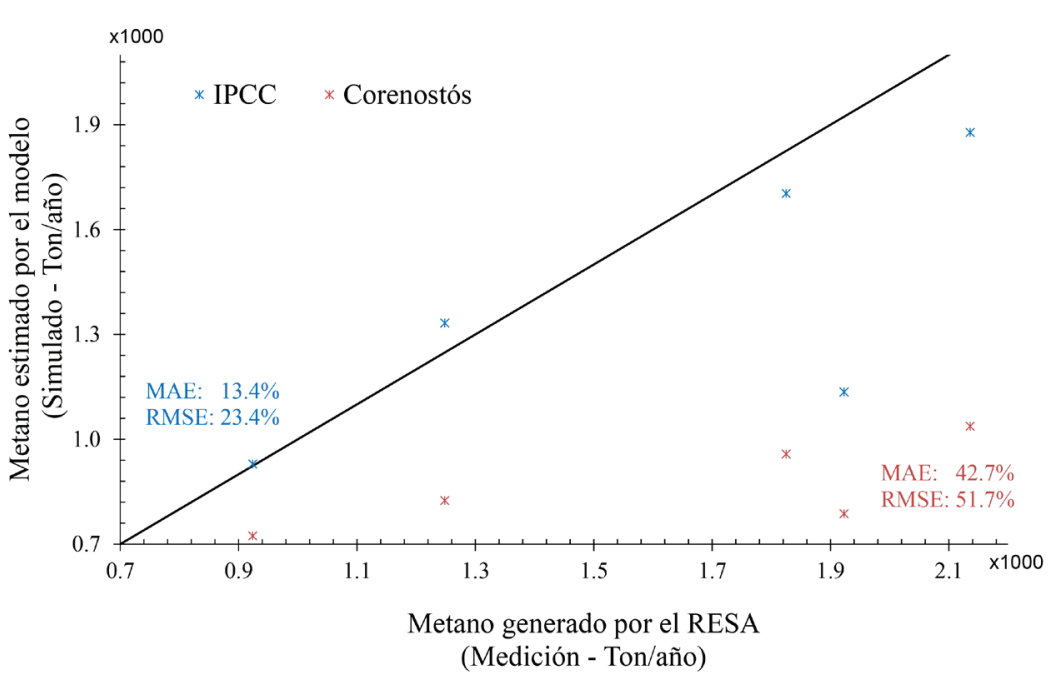

Figura 4. Comparación entre las estimaciones de $\mathrm{CH}_{4}$ de los modelos y los valores medidos.

MAE: Error medio absoluto. RMSE: Raíz del error cuadrático medio dumbre, utilizar información más puntal y precisa en cuanto a la variación de la composición física de los residuos, parámetros de funcionamiento del RESA, las características químicas, el índice de generación de metano entre otros factores.

\section{Conclusiones}

La ciudad de Santa Marta presenta una ppc de residuos ligeramente superior a las condiciones del contexto colombiano con una tasa de crecimiento equivalente a la poblacional nacional. Igualmente, la dinámica mensual de segregación, así como su composición de los residuos con predominancia de orgánicos y alto contenido de humedad, se asocia a la influencia que ejerce su vocación turística. Las estimaciones de metano por los modelos se consideran entre aceptables (IPCC) y deficientes (Corenostós). Sin embargo, se observa una subestimación por ambos modelos de las emisiones, donde el modelo del IPCC es el de mejor ajuste para estimar la producción de biogás en el relleno.

\section{AgradeCimientos}

A la ingeniera Eliana Álvarez, Funcionaria de la Corporación Autónoma Regional del Magdalena (CORPAMAG), por el respaldo brindado en la consecución de la información con la que se realizó la investigación.

\section{Referencias}

Aguilar-Virgen Q., Ojeda-Benítez S., Taboada-González P., Quintero-Núñez M. Estimación de las constantes k y L0 de la tasa de generación de biogás en sitios de disposición final en Baja
California, México. Rev Int Contam Ambient, volumen 28, 2012: 45-51.

Aguilar-Virgen Q., Taboada-González P.A., Ojeda-Benítez S. Potential production of electricity from biogas generated in a sanitary landfill. Ingeniería e Investigación, volumen 31, 2011a: 56-65.

Aguilar-Virgen Q., Taboada-González P.A., Ojeda-Benítez S. Modelo mexicano para la estimación de la generación de biogás. Ingeniería Revista Académica, volumen 15, 2011b: 37-45.

Alcaldía de Santa Marta, Interconexión Eléctrica ISA, \& Programa de las Naciones Unidad para el Desarrollo PNUD, Estado de avance de los objetivos del milenio, Alcaldía de Santa Marta, Santa Marta, Colombia, 2012.

Aluna consultores limitada. Estudio nacional del reciclaje y los recicladores: Historia del reciclaje y los recicladores de Colombia, Colombia, 2011.

Aronica S., Bonanno A., Piazza V., Pignato L., Trapani S. Estimation of biogas produced by the landfill of Palermo, applying a Gaussian model. Waste Management, volumen 29, 2009: 233239. doi: 10.1016/j.wasman.2008.02.026

Arrieta G.B. Análisis de la producción de residuos sólidos de pequeños y grandes productores, determinación de factores de producción de residuos sólidos de los usuarios residenciales, revisión de la regulación vigente y cálculo de costos asociados a la realización de aforo de residuos sólido en Colombia. Comisión de Regulación de Agua y Saneamiento Básico-(CRA)-Ministerio de Ambiente, Viviendo y Desarrollo Territorial, Colombia, 2008.

Börjesson G., Samuelsson J., Chanton J., Adolfsson R., Galle B., Svensson B.H. A national landfill methane budget for Sweden based on field measurements, and an evaluation of IPCC models. Tellus B., 2009. doi: 10.3402/tellusb.v61i2.16841 
Camargo Y., Henao D.M., Vélez-Pereira A.M. Emisiones atmosféricas de origen biológico, Primera, Colombia, Fondo Editorial UniMagdalena, Santa Marta, 2011.

Camargo Y., y Vélez-Pereira A.M. Emisiones de biogás producidas en rellenos sanitarios, en: Proceedings La Gestión Sostenible de los Residuos, Barranquilla, Colombia, 2009, p. 50.

Cendales LED. Producción de biogás mediante la codigestión anaeróbica de la mezcla de residuos cítricos y estiércol bovino para su utilización como fuente de energía renovable (tesis para Magíster en Ingeniería Mecanica), Universidad Nacional de Colombia, 2011.

Collazos H. Diseño y operación de rellenos sanitarios, 2da ed., Escuela Colombia de Ingeniería, Bogotá, 2005.

Ducom G., Radu-Tirnoveanu D., Pascual C., Benadda B., Germain P. Biogas-Municipal solid waste incinerator bottom ash interactions: Sulphur compounds removal. Journal of Hazardous Materials, volumen 166, 2009: 1102-1108. doi: 10.1016/j.jhazmat.2008.12.024

ESPA. Plan de gestión integral de los residuos sólidos del Distrito de Santa Marta, volumen II, Diagnóstico integral del PGIRS, ESPA, Santa Marta, Colombia, 2014.

Espíritu-Barragán C., Vera-Romero I., Estrada-Jaramillo M., Ortiz-Soriano A., Medina-Orozco L., Martínez-Reyes J. Estimación de biogás como base para la implementacion de un relleno sanitario intermunicipal: Jiquilpan, Sahuayo y Venustiano Carranza, en: Paz-Rellat F. y Wong-González J. (eds) Estado actual del conocimiento del ciclo del Carbono y sus interacciones en México: Síntesis a 2013, 1st ed., Programa Mexicano del Carbono, Texcoco, Estado de México, México, 2014, pp. 669-674.

Garrido G.A.P. y Camargo C.Y. Distribución del espacio temporal de partículas suspendidas totales en la zona costera del departamento del Magdalena en el periodo 2006-2009. Revista de Ciencias, volumen 18, 2015: 59-72.

Georgaki I., Soupios P., Sakkas N., Ververidis F., Trantas E., Vallianatos F., Manios T. Evaluating the use of electrical resistivity imaging technique for improving $\mathrm{CH}_{4}$ and $\mathrm{CO}_{2}$ emission rate estimations in landfills. Science of The Total Environment, volumen389, 2008:522-531. doi:10.1016/j.scitotenv.2007.08.033

Guerra P.E. Simulación de modelos de sistemas dinámicos para sostenibilidad, (tesis de ingeniería informatica), Universitat Politècnica de Catalunya, 2009.

Hilkiah-Igoni A., Ayotamuno M.J., Eze C.L., Ogaji S.O.T., Probert S.D. Designs of anaerobic digesters for producing biogas from municipal solid-waste. Applied Energy, volumen 85, 2008: 430438. doi: 10.1016/j.apenergy.2007.07.013

Hoornweg D. y Bhada-Tata P. What a waste: A global review of solid waste management, World Bank, Washington, 2012.

Im J., Moon S., Nam K., Kim Y-J., Kim J.Y. Estimation of mass transport parameters of gases for quantifying $\mathrm{CH} 4$ oxidation in landfill soil covers. Waste Manage, volumen 29, 2009: 869875. doi: 10.1016/j.wasman.2008.07.006
IPCC, Panel Intergubernamental de Cambio Climático, directrices del IPCC para los inventarios de gases de efecto invernadero, IPCC, 1996.

IPCC, Panel Intergubernamental de Cambio Climático Waste., en: Good Practice Guidance and Uncertainty Management in National Greenhouse Gas Inventories, Institutefor Global Environmental Strategies (IGES), Japón, 2002, pp 419-468.

IPCC, Panel Intergubernamental de Cambio Climático, Eggleston H.S., Buendia L., Miwa K., Ngara T. y Tanabe K. (eds) Waste, en: Guide lines for National Greenhouse Gas Inventories. IGES, Japón, 2006.

Jaramillo G. y Zapata L. Aprovechamiento de los residuos sólidos orgánicos en Colombia (tesis para optar al título de Especialista en Gestión Ambiental), Universidad de Antioquia, 2008.

Kalantarifard A. y Yang G.S. Estimation of methane production by LANDGEM simulation model from tanjung langsat municipal solid waste landfill, Malaysia. International Journal of Science and Technology, volumen 1, 2012: 481-487.

Kim H-S. y Yi S-M. Methane emission estimation from landfills in Korea (1978-2004): quantitative assessment of a new approach. J Air Waste Manag Assoc, volumen 59, 2009: 70-77.

Lobo A. y Tejero I. Application of simulation models to the diagnosis of MSW landfills: An example. Waste Manage, volumen 27, 2007: 691-703. doi: 10.1016/j.wasman. 2006.04.015

López C.C. Emisiones de metano derivadas de los desechos sólidos municipales en Cuba. Observatorio Medioambiental, volumen 14, 2011: 279-300.

Mackie K.R. y Cooper C.D. Landfill gas emission prediction using Voronoi diagrams and importance sampling. Environmental Modelling \& Software, volumen 24, 2009: 1223-1232. doi: 10.1016/j.envsoft.2009.04.003

Mann H.B. y Whitney D.R. On a test of whether one of two random variables is stochastically larger than the other, The annals of mathematical statistics, 1947, pp. 50-60.

Martín G.S. Producción y recuperación de biogás en vertederos controlados de residuos sólidos urbanos: análisis de variables y modelización, Universidad de Oviedo, 1997.

Mejía C.A.Z. y Gil V.H.G. Análisis de la producción de lixiviado y biogás bajo condiciones de extracción activa en un relleno sanitario. Ingenium, volumen 16, 2015: 9-23. doi: http://dx.doi. org/10.21500/01247492.1365

Meraz R-L., Vidales A-M., Domínguez A. A fractal-like kinetics equation to calculate landfill methane production. Fuel, volumen 83, 2004: 73-80. doi: 10.1016/S0016-2361(03)00212-6

Ministerio de Vivienda, Ciudad y Territorio y Viceministerio de Agua y Saneamiento Básico, Reglamento técnico del sector de agua potable y Saneamiento básico, Título F, Sistemas de Aseo Urbano, 2012.

Monsalve S.G. Hidrología en la ingeniería, 2a ed., Mexico, Alfaomega grupo editor, 1999. 
DOI: http://dx.doi.org/10.22201/fi.25940732e.2017.18n2.016

Armenta-Rivas Maysson Esmi, Sierra-Camargo liseth Dayana, Vélez-Pereira Andrés M.

Murphy J.D. y McKeogh E. The benefits of integrated treatment of wastes for the production of energy. Energy, volumen 31, 2006: 294-310. doi: 10.1016/j.energy.2005.02.003

Observatorio de Políticas Públicas de Medellín, Boletín informativo medio ambiente, Alcaldía de Medellín, Departamento Administrativo de Planeación, Medellín, 2010.

Panesso A.F., Cadena J.A., Flórez J.J.M., Ordoñez M. del C. Análisis del biogás captado en un relleno sanitario como combustible primario para la generación de energía eléc. Scientia et Technica, volumen 1, 2011: 23-28.

Rada E.C., Ragazzi M., Stefani P., Schiavon M., Torretta V. Modelling the Potential Biogas Productivity Range from a MSW Landfill for Its Sustainable Exploitation. Sustainability, volumen 7, 2015: 482-495. doi: 10.3390/su7010482

Rojas C.A., Rodríguez B.R., Álamo H.U., Pacheco M.L.E., Treviño S.S., Márquez S.M. Experiencia de participación comunitaria para el manejo adecuado de residuos sólidos urbanos en México, Global Health Promotion 1757975914543576, 2014. doi: 10.1177/1757975914543576

Schmidt F. Valoración del biogás en un relleno sanitario, en: Seminario Internacional Gestión Integral de Residuos Sólidos y Peligrosos, Siglo XXI, Medellín, Colombia, 1999, pp. 1-11

SCS Engineers Evaluación del relleno sanitario y estudio de prefactibilidad para la recuperación y utilización de biogás en el relleno sanitario El Navarro Cali, Colombia, SCS Engineers, Reston, Virginia, 2008.

SCS Engineers Estudio de pre-factibilidad para la recuperación de biogás y producción de energía relleno sanitario El Carrasco Bucaramanga, Colombia, SCS Engineers, Reston, Virginia, 2005.

Serrano C. Alternativas de utilización de biogás de rellenos sanitarios en Colombia (tesis para Magister en Gestión y auditorías Ambientales), Universidad de las Palmas de Gran Canaria, 2006.

Sierra S., Alberto C., Barrios A., Leonor R., Morales J., Carmen J.D. Landfill mining as an alternative for solid waste management. Producción + Limpia, volumen 9, 2014: 115-123.
Singh R.P., Tyagi V.V., Allen T., Ibrahim M.H., Kothari R. An overview for exploring the possibilities of energy generation from municipal solid waste (MSW) in Indian scenario. Renew Sust Energ Rev, volumen 15, 2011: 4797-4808. doi: 10.1016/j. rser.2011.07.071

SSPD Superintendencia de servicios públicos (2011). Situación de la disposición final de residuos sólidos en Colombia- diagnostico, 2011.

Terraza H. Manejo de residuos sólidos: Lineamientos para un servicio integral, sustentable e inclusivo, Nueva York, Inter-American Development Bank, 2009.

Urrego-Martínez E., Rodríguez-Miranda J.P., Sánchez-Céspedes J.M. Application of a proposed methodology for estimating natural gas in a landfill. Case study landfill Juana, Bogota, Colombia. Int J Appl Environ Sci, volumen 11, 2016:1153-1172.

Vélez-Pereira A., Caicedo Y.C., Rincones S.R.B. Distribución espacio-temporal de aerobacterias en el relleno sanitario Palangana, Santa Marta (Colombia). INTROPICA, volumen 5, 2010: 5-18.

Vera-Romero I., Estrada-Jaramillo M., Martínez-Reyes J., OrtizSoriano A. Potencial de generación de biogás y energía eléctrica. Parte II: residuos sólidos urbanos. Ingeniería, Investigación y Tecnología, volumen 16, 2015: 471-478. doi: 10.1016/j. riit.2015.05.012

Vera-Romero I., Reyes J.M., Espíritu-Barragán C.C., Estrada-Jaramillo M., Ortiz-Soriano A., Medina-Orozco L.E. Biogas Estimation as a Basis for the Implementation of an Intermunicipal Landfill: Michoacan, Mexico. Journal of Environmental Protection, volumen 5, 2014: 577-582. doi: 10.4236/jep.2014.57059

Wangyao K., Yamada M., Endo K., Ishigaki T., Naruoka T., Towprayoon S., Sutthasil N. Methane generation rate constant in tropical landfill. Journal of Sustainable Energy \& Environment, volumen 1, 2010: 181-184.

Wilcoxon F. Individual comparisons by ranking methods. Biometrics bulletin, 1945: 80-83.

Zamorano M., Pérez-Pérez I.J., Aguilar-Pavés I., Ramos-Ridao Á. Study of the energy potential of the biogas produced by an urban waste landfill in Southern Spain. Renew Sust Energ Rev, volumen 11, 2007: 909-922. doi: 10.1016/j.rser.2005.05.007 
DOI: http://dx.doi.org/10.22201/fi.25940732e.2017.18n2.016

Modelación de la producción de metano en el Relleno Sanitario Parque Ambiental Palangana (Santa Marta)

\section{Citación sugerida:}

\section{Citación estilo Chicago}

Armenta-Rivas, Maysson Esmi, Liseth Dayana Sierra-Camargo, Andrés M. Vélez-Pereira. Modelación de la producción de metano en el ReIleno Sanitario Parque Ambiental Palangana (Santa Marta). Ingeniería Investigación y Tecnología, XVIII, 02 (2017): 183-192.

\section{Citación estilo ISO 690}

Armenta-Rivas M.E, Sierra-Camargo L.D., Vélez-Pereira A.M. Modelación de la producción de metano en el Relleno Sanitario Parque Ambiental Palangana (Santa Marta). Ingeniería Investigación y Tecnología, volumen XVIII (número 2), abril-junio 2017: 183-192.

\section{Semblanzas de los autores}

Maysson Esmi Armenta-Rivas. Realizó sus estudios profesionales en Ingeniería Ambiental y Sanitaria en la Universidad del Magdalena (Santa Marta), pertenece al Grupo de Investigación en Modelación de Sistemas Ambientales (GIMSA). Desde el año 2014 se encuentra laborando para la compañía del sector alimenticio C.I FAMAR S.A. liderando el proceso de manejo ambiental.

Liseth Dayana Sierra-Camargo. Realizó sus estudios profesionales en Ingeniería Ambiental y Sanitaria en la Universidad del Magdalena (Santa Marta), pertenece al Grupo de Investigación en Modelación de Sistemas Ambientales (GIMSA).

Andrés M. Vélez-Pereira. Ingeniero Ambiental y Sanitario y Especialista en Gestión Ambiental por la Universidad del Magdalena, Magister en Tecnología Ambiental por la Universidad Internacional de Andalucía y la Universidad de Huelva. Doctorando en Ciencia y Tecnología Ambientales por la Universidad Autónoma de Barcelona. Cuenta con experiencia en docencia e investigación en procesos biológicos, contaminación atmosférica y meteorología. 